\title{
Haplotype analysis of the CAG and CCG repeats in 21 Brazilian families with Huntington's disease
}

\author{
This article has been corrected since Advance Online Publication, and an erratum is also printed in this issue.
}

\author{
Luciana de A Agostinho ${ }^{1,2}$, Catielly F Rocha ${ }^{1}$, Enrique Medina-Acosta ${ }^{3}$, Hazel N Barboza ${ }^{3}$, \\ Antônio F Alves da Silva ${ }^{3}$, Simão PF Pereira ${ }^{2}$, Iane dos Santos da Silva ${ }^{4}$, Eduardo R Paradela ${ }^{1}$, \\ André L dos S Figueiredo ${ }^{1}$, Eduardo de M Nogueira ${ }^{4}$, Regina MP Alvarenga ${ }^{1}$, Pedro Hernan Cabello ${ }^{5,6}$, \\ Suely R dos Santos ${ }^{4}$ and Carmen LA Paiva ${ }^{1,4}$
}

We studied the allelic profile of CAG and CCG repeats in 61 Brazilian individuals in 21 independent families affected by Huntington's disease (HD). Thirteen individuals had two normal alleles for HD, two had one mutable normal allele and no HD phenotype, and forty-six patients carried at least one expanded CAG repeat allele. Forty-five of these individuals had one expanded allele and one individual had one mutable normal allele (27 CAG repeats) and one expanded allele (48 CAG repeats). Eleven of these forty-five subjects had a mutant allele with reduced penetrance, and thirty-four patients had a mutant allele with complete penetrance. Inter- and intragenerational investigations of CAG repeats were also performed. We found a negative correlation between the number of CAG repeats and the age of disease onset $(r=-0.84 ; P<0.001)$ and no correlation between the number of CCG repeats and the age of disease onset $(r=0.06)$. We found 40 different haplotypes and the analysis showed that $(C C G)_{10}$ was linked to a CAG normal allele in 19 haplotypes and to expanded alleles in two haplotypes. We found that (CCG) 7 was linked to expanded CAG repeats in 40 haplotypes $(95.24 \%$ ) and (CCG) 10 was linked to expanded CAG repeats in only two haplotypes (4.76\%). Therefore, $(\mathrm{CCG})_{7}$ was the most common allele in $\mathrm{HD}$ chromosomes in this Brazilian sample. It was also observed that there was a significant association of $(C C G)_{7}$ with the expanded CAG alleles $\left(\chi^{2}=6.97, P=0.0084\right)$. Worldwide, the most common CCG alleles have 7 or 10 repeats. In Western Europe, (CCG) $)_{7}$ is the most frequent allele, similarly to our findings.

Journal of Human Genetics (2012) 57, 796-803; doi:10.1038/jhg.2012.120; published online 11 October 2012

Keywords: CAG repeats; CCG repeats; Huntington's disease

\section{INTRODUCTION}

Huntington's disease (HD) is a rare, progressive and fatal neurodegenerative disease characterized by a lack of motor coordination of both voluntary and involuntary muscles (choreic movements), personality disorders and progressive dementia., ${ }^{1,2} \mathrm{HD}$ usually manifests in patients between 35 and 55 years of age, ${ }^{3,4}$ and average life expectancy is 15 to 20 years after the onset of the disease. The prevalence of HD varies with ethnicity. HD prevalence in Asian countries, such as Japan and China, is below that in Caucasian populations of Western Europe. ${ }^{5,6}$

HD results from the mutation of the Huntingtin (HTT) gene, which is located on the short arm of chromosome 4 (4p16.3). The mutation occurs as a variation in the number of CAG repeats, located in the first exon of gene HTT, which encodes a polyglutamine track next to the amino-terminal region of the HTT protein. Normal alleles have fewer than 27 copies of the CAG trinucleotide. Mutable normal alleles have from 27 to 35 CAG repeats. The alleles with 36 to 39 CAG repeats have reduced penetrance and those with more than 39 copies of CAG show complete penetrance. HTT is expressed throughout the body in neuronal and non-neuronal cells. ${ }^{7,8}$ Interestingly, the supposed gain of toxic function of mutated HTT reaches only certain neural regions, such as the striatum and cerebral cortex. Despite many extensive experimental investigations, there are no prophylactic or curative treatments for HD. ${ }^{9}$

The aims of this study were to investigate, at the molecular level, the CAG/CCG haplotypes in Brazilian individuals affected by HD and associated risk groups, to determine whether the number of CAG and CCG repeats correlate with the age of onset of symptoms and to perform intra- and intergenerational investigation of the CAG repeats.

Importantly, the investigation of the CCG region and CAG/CCG haplotypes is novel because it focuses on the previously

${ }^{1}$ Programa de Pós-Graduação em Neurologia, UNIRIO, Rio de Janeiro, Brazil; ${ }^{2}$ Department of Biomedicine, Faculdade de Medicina, FAMINAS, Muriaé, Brazil; ${ }^{3}$ Núcleo de Diagnóstico e Investigação Molecular, Universidade Estadual do Norte Fluminense Darcy Ribeiro, Campos dos Goytacazes, Brazil; ${ }^{4}$ Department of Genetics and Molecular Biology, Universidade Federal do Estado do Rio de Janeiro (UNIRIO), Rio de Janeiro, Brazil; ${ }^{5}$ Laboratório de Genética Humana, FIOCRUZ, Rio de Janeiro, Brazil and ${ }^{6}$ Laboratório de Genética Humana, UNIGRANRIO, Duque de Caxias, Brazil

Correspondence: Professor CLA Paiva, Department of Genetics and Molecular Biology, Universidade Federal do Estado do Rio de Janeiro (UNIRIO), Rua Custodio Serrao 14/ 701, Rio de Janeiro, RJ CEP 22470-230, Brazil.

E-mail: clapaiva1@gmail.com

Received 30 March 2012; revised 28 August 2012; accepted 4 September 2012; published online 11 October 2012 
uncharacterized Brazilian population. Furthermore, the ancestral origin(s) of the mutated gene in Brazilian patients has not been established and our results indicate that HTT mutations likely have more than one ancestral origin.

\section{MATERIALS AND METHODS \\ Patient sampling}

This is a descriptive transversal study of HD in Brazil. Sixty-one individuals, representing 21 independent families, participated in the survey. Participants were recruited from the Gaffrée and Guinle University Hospital outpatient Genetics Clinic (in Rio de Janeiro), the Huntington's disease Association in Brazil (members from the state of Rio de Janeiro) and the city of Ervália, in the state of Minas Gerais. All subjects were Brazilians from the South-Eastern Region, including the states of Rio de Janeiro (45.9\%), Minas Gerais (39.3\%), Espírito Santo (11.5\%) and São Paulo (3.3\%).

This study was approved by the Ethics in Research Committees of Gaffree and Guinle University Hospital/UNIRIO (number 03/2009) and FAMINASMuriae-MG (number 100331/0003). Subjects were included in the study if they presented clinical signs and symptoms typical of HD, as confirmed by a neurologist, and/or were considered at risk for developing HD. It is important to mention that those subjects who had an affected mother or father were considered to be at risk. All participating individuals or their legal guardians had to fill in and sign an informed consent form. Foreign and adopted individuals were excluded from the survey. A questionnaire was used to collect the participant data. The pedigree for each family was produced using the 2007 version of the GenoPro program (free online at http://www.genopro.com/) (results not shown). The HD alleles were classified by repeat length, according to the ACMG/ASHG statement. ${ }^{10}$ It is important to mention that the data collected from normal subjects, who had only normal alleles $(<27$ CAG repeats), were used to construct pedigrees and calculate the frequency of each CAG allele (122 total alleles in 61 subjects). The data from normal individuals were excluded from the sample for further statistical analysis.

\section{Genotyping}

Genomic DNA was extracted from either peripheral blood (1-3 ml in EDTA) or mouth epithelial cells (swabs) using the Illustra blood genomic Prep Mini Spin kit (GE Healthcare, Little Chalfont, UK).

\section{CAG and CAG-CCG analysis}

Three primers (6 FAM - 5' -TGGCGACCCTGGAAAAGCTGAT-3' (forward 1, HD1), 5'-GCGGTGGCGGCTGTTGCTGCT-3' (reverse 1, HD3) and 5'-CGG CGGCGGCTGAGGAAGCTG-3' (reverse 2, HD4)) were used to amplify the target DNA (CAG and CAG + CCG polymorphic regions). PCR reactions contained 0 to $100 \mathrm{ng}$ of DNA, $10 \mathrm{pmols}$ of each primer, $6.25 \mu \mathrm{l}$ of Promega GoTaq Green Master Mix (containing $1.5 \mathrm{~mm} \mathrm{MgCl}_{2}$ and $200 \mu \mathrm{M}$ of each dNTP) and sufficient DNAse-free water to make the final volume (12.5 $\mu \mathrm{l})$ of each reaction. Two distinct products were PCR amplified; one of the products was used to analyse the polymorphic CAG repeat region and the other product was used to analyse both the CAG and CCG repeat regions on phase. Thermal cycling conditions were $94^{\circ} \mathrm{C}$ for $5 \mathrm{~min} ; 35$ cycles of $94^{\circ} \mathrm{C}$ for $1 \mathrm{~min}, 59.1^{\circ} \mathrm{C}$ for $1 \mathrm{~min}$ and $72^{\circ} \mathrm{C}$ for $2 \mathrm{~min}$; and $72^{\circ} \mathrm{C}$ for $50 \mathrm{~min}$. Allele profiles were determined on an ABI 3500 Genetic Analyser (Applied Biosystems, Foster City, CA, USA). The data were analysed using the GeneScan Analysis 3.7 and Genotyper 3.7 programs (Applied Biosystems).

\section{CCG analysis}

To genotype the CCG polymorphic region alone, and thus to validate the CAG-CCG analysis above, the following pair of primers was used (6 FAM - 5'AACAGCCGCCACCGCCGC-3' (forward 2, D4S3076662F) and 5'-GCGGCT GAGGCAGCAGCGG-3' (reverse 3, D4S3076748R)). PCR reactions contained $2 \mathrm{ng}$ of DNA, $10 \mathrm{pmols}$ of each primer, $6.25 \mu \mathrm{l}$ of Master Mix (containing $1.0 \mathrm{~mm} \mathrm{MgCl} 2$ and $200 \mu \mathrm{m}$ of each dNTP) (Invitrogen, Life Technologies, Grand Island, NY, USA) and sufficient DNAse-free water to make the final volume $(12.5 \mu \mathrm{l})$ of each reaction. Thermal cycling conditions were: $94^{\circ} \mathrm{C}$ for $5 \mathrm{~min} ; 35$ cycles of $94^{\circ} \mathrm{C}$ for $1 \mathrm{~min}, 72^{\circ} \mathrm{C}$ for $3 \mathrm{~min}$ (the same temperature was used for primer annealing and extension); and extension was completed at $72{ }^{\circ} \mathrm{C}$ for $10 \mathrm{~min}$. Allele profiles were determined on an ABI 3500 Genetic Analyser (Applied Biosystems). The data were analysed using the GeneScan Analysis 3.7 and Genotyper 3.7 programs (Applied Biosystems). Therefore, the sizes of CCG polymorphic regions were determined by two methods: the direct method using a pair of primers that spanned only CCG region (D4S3076662F and $\mathrm{D} 4 \mathrm{~S} 3076748 \mathrm{R}$ ) and the indirect one using three different primers that spanned CAG region (HD1 and HD3) and CAG + CCG region (HD1 and HD4).

\section{Statistical analysis}

Univariate analysis of the data was performed. Mean ( \pm standard deviation), median and proportions were calculated using the IBM SPSS Statistics software (version 14.0, Somers, NY, USA). The correlation between two variables was measured by 'Pearson's $r$ '. Association between CCG alleles and size of CAG allele was performed using Woolf's method ${ }^{11}$ with correction according to Haldane. ${ }^{12}$

\section{RESULTS}

We investigated the haplotypes of 61 individuals who were affected by HD or were at risk for HD from April 2009 to March 2011. Twentyone pedigrees were created using data collected during interviews. These 21 pedigrees included a total of 534 individuals; 197 individuals (36.9\%) were clinically affected by $\mathrm{HD}$ and 337 (63.1\%) were classified as at risk because they were the offspring of one affected parent and, therefore, had a $50 \%$ chance of inheriting the diseasecausing mutant gene.

The flowchart in Figure 1a shows the genotypes (number of CAG repeats) and the phenotypes at the time of the survey. The places of birth and residence of each subject with an abnormal HD allele are shown in panels b and c, respectively. All of the patients $(n=46)$ were Brazilian, and they were born in the states of Rio de Janeiro (50\%), Minas Gerais (32.6\%), Espírito Santo (15.2\%) and São Paulo (2.2\%). At the time of the survey, 28/46 (60.9\%) of these subjects lived in Rio de Janeiro, and 18/46 (39.1\%) lived in Minas Gerais.

The age of onset of the disease varied from 18 to 56 years, with a mean of $41.7 \pm 10.1$ years and a median of 43 years. At the time of our investigation, only 5 of the 46 patients with at least one expanded CAG repeat allele had no signs or symptoms of the disease. In $55.3 \%$ of the patients, the disease started before 49 years of age. One case of juvenile disease was found. That person had 49 CAG repeats and first showed symptoms of HD when he was 18 years old. Statistical analysis of the data showed a strong negative correlation (Pearson's $r=-0.84 ; P<0.001$ ) between the age of onset of the disease and the number of CAG repeats (Figure 2).

\section{CAG alleles}

Based on the molecular analysis, 13 subjects $(21.31 \%$ of the total sample) were negative for CAG expansion consistent with $\mathrm{HD}$ and were thus classified as normal (Figure 1a). Two of the normal individuals had a mutable normal allele (27 CAG repeats).

On the other hand, 46 of the participants had at least one expanded CAG repeat allele. One of the forty-six patients had one mutable normal allele (27 CAG repeats) and one allele of complete penetrance (48 CAG repeats). Interestingly, this individual was born in Ervália, a city with a high prevalence of HD (results not shown). Her symptoms started when she was 28 years old. By contrast, another patient, who had the same mutant allele (48 CAG repeats) and one normal allele (14 CAG repeats), did not become symptomatic until he was 35 years old.

Of the 46 individuals with an expanded allele, 20 were male and 26 were female. Paternal and maternal inheritance of the expanded alleles 


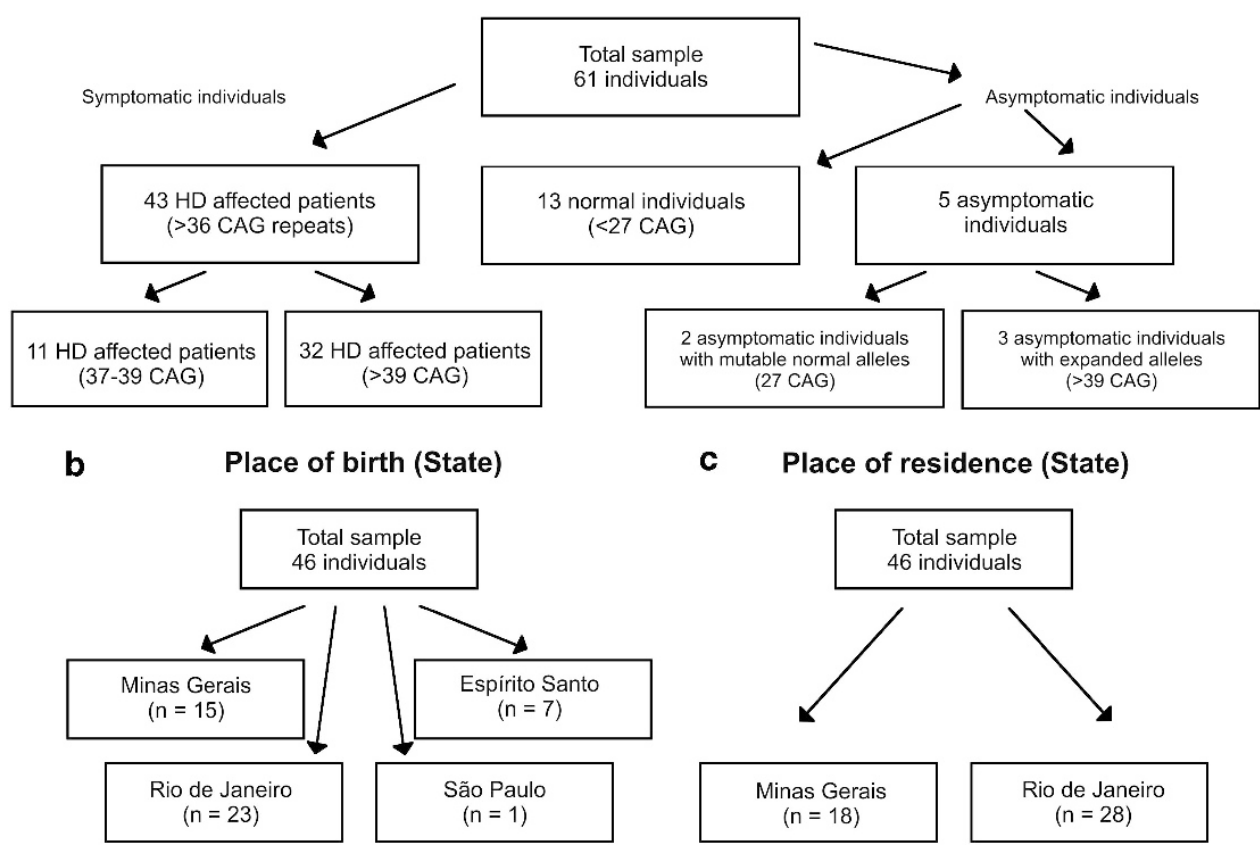

Figure 1 (a) Genotypes (number of CAG repeats) and phenotypes: normal (clinically and molecularly normal), affected by the disease or asymptomatic at the time of the survey. (b) Location (state) of birth. (c) Location (state) of residence.

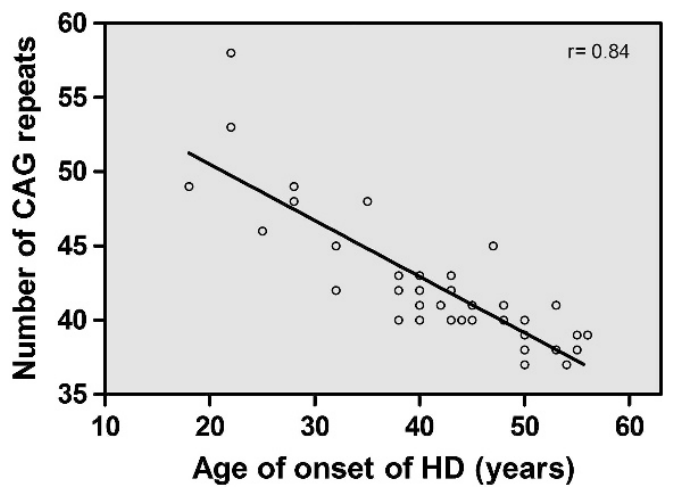

Figure 2 Correlation between the number of CAG repeats and the age of onset of HD.

occurred in $55.3 \%$ and $44.7 \%$ of the cases, respectively, based on the pedigree information and the molecular HD testing.

The length of the CAG expansion varied from 12 to 58 repeats in the 122 alleles tested. The frequency of each allele in our sample is shown in Figure 3. Among 13 subjects with only normal alleles, 4 were homozygous for an allele bearing 15 CAG repeats.

In the heterozygous subjects $(n=46)$, who each had at least one expanded allele, the normal alleles contained 12 to 26 CAG repeats, with a mean of $16 \pm 3.3$ and a median of 15 . No subjects were homozygous for an expanded CAG repeat allele. As mentioned before, only one of the subjects had one completely penetrant expanded allele and one mutable normal allele.

In this study, the mutable normal alleles had 27 CAG repeats, and the expanded alleles had 37 to 58 CAG repeats (mean value $42.5 \pm 4.4$ ). In $50 \%$ of the cases, the expanded allele had 41 or more CAG repeats. In the sample group, 2 individuals (4.1\%) had mutable normal alleles bearing 27 CAG repeats; $11(23.0 \%)$ had alleles of reduced penetrance ( 37 to $39 \mathrm{CAG}$ repeats); and 35 (70.8\%) had alleles of complete penetrance ( $>39$ CAG repeats). The shortest expanded allele that caused a disease phenotype had 37 CAG repeats, and the longest had 58 repeats. At the time of the investigation, three carriers of completely penetrant alleles had not shown any signs or symptoms of HD. On the other hand, all of the carriers of a reduced penetrance allele showed signs or symptoms of the disease.

\section{CCG alleles}

Another polymorphic region containing CCG trinucleotides is found 12 bp away from the CAG region. This region was also analysed in the 21 families described above. The number of CCG repeats of HD chromosomes varied from 6 to 10 (mean 7.5 1.2) (Figure 4). The sizes of CCG polymorphic regions were determined by two methods as described in Materials and methods. The results of CCG genotyping with both strategies were consistent.

Repeats of seven CCG trinucleotides were found in $78.7 \%$ of the sample. The CCG alleles linked to the expanded CAG alleles, had 6, 7, 8 or 10 CCG repeats (mean $7.1 \pm 0.7$ ), and $40 / 46(86.9 \%$ ) of these alleles had seven $\mathrm{CCG}$ repeats $(\mathrm{CCG})_{7}$. Statistical analysis of this data showed that there is no correlation (Pearson's $r=0.06 ; P<0.001$ ) between age of onset of the disease and the number of CCG repeats. Eighteen of the twenty-one families had CCG tracks of the same size; fifteen had seven CCG repeats $(\mathrm{CCG})_{7}$, one had eight repeats $(\mathrm{CCG})_{8}$, and two had ten repeats (CCG) 10 . Three of the twenty-one families had different CCG alleles within the family; two families had (CCG) and $(\mathrm{CCG})_{7}$ alleles, and one family had $(\mathrm{CCG})_{7}$ and $(\mathrm{CCG})_{8}$. Of the families that lived in Rio de Janeiro state $(n=17), 13$ had $(\mathrm{CCG})_{7}, 3$ had $(\mathrm{CCG})_{10}$ and 1 had $(\mathrm{CCG})_{8}$. The four families from Ervalia had $(\mathrm{CCG})_{7}$ alleles, but three of them had at least one member who carried a CCG allele with \pm 1 CCG repeat. 


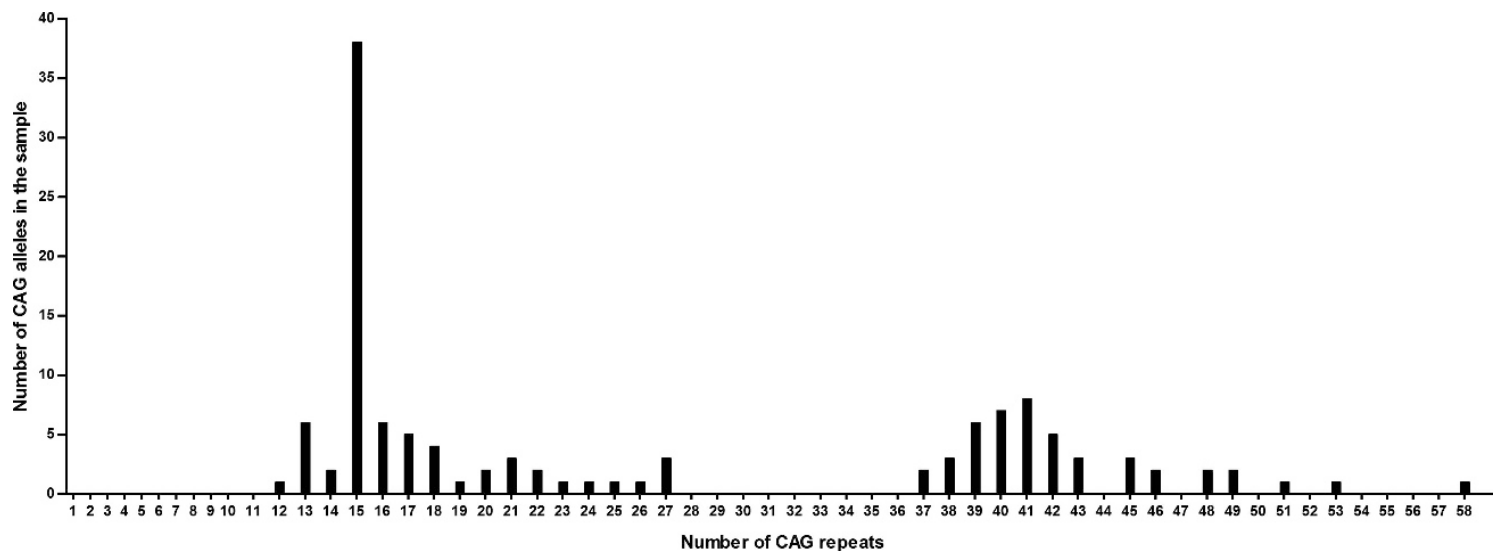

Figure 3 Number of CAG alleles in the sample ( $n=122$ alleles) versus number of CAG repeats in each allele.

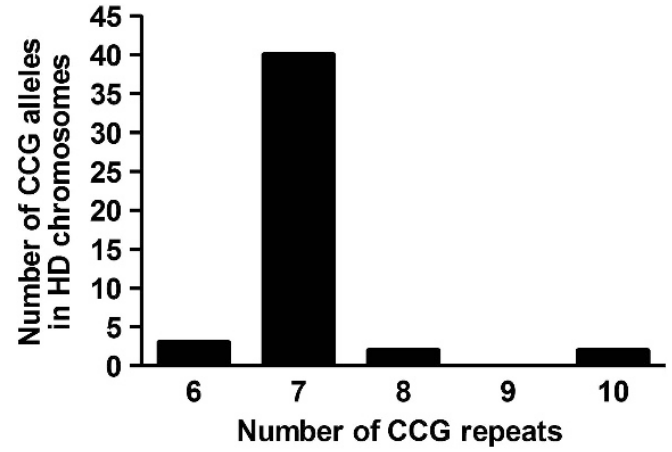

Figure 4 Number of CCG alleles $(n=46)$ in HD chromosomes versus number of CCG repeats in each allele.

\section{Haplotype analysis}

DNA haplotypes were determined using primers that spanned tracts of CAG and CCG trinucleotides. One pair of primers that spanned only the CCG repeats was used to validate the results. The sizes of both the CAG and the CCG repeats in the HTT gene and frequency of each allele were analyzed in normal and HD chromosomes. Altogether, 40 different haplotypes were found in the 122 analyzed chromosomes (Table 1). Among the normal and the HD chromosomes, 22 and 18 different haplotypes were found, respectively. The most common haplotype of $\mathrm{HD}$ chromosomes was $(\mathrm{CAG})_{40}$ $(\mathrm{CCG})_{7}$, which represents $15.22 \%$ of the HD chromosomes. Another common haplotype was $(\mathrm{CAG})_{39}-(\mathrm{CCG})_{7}$, which occurred in $13.04 \%$ of the HD chromosomes. The $(\mathrm{CAG})_{42}-(\mathrm{CCG})_{7}$ haplotype was observed in $10.87 \%$ of the HD chromosomes. Analysis of the CAG-CCG haplotypes revealed that the HD-expanded CAG repeat alleles were linked to alleles with $6,7,8$ or 10 copies of the repeat. Specifically, 3, 40, 1 and 2 HD-expanded CAG repeat alleles were linked with $(\mathrm{CCG})_{6},(\mathrm{CCG})_{7},(\mathrm{CCG})_{8}$ and $(\mathrm{CCG})_{10}$, respectively. In normal chromosomes, the $(\mathrm{CAG})_{15}-(\mathrm{CCG})_{7}$ and $(\mathrm{CAG})_{15}-$ (CCG) ${ }_{10}$ haplotypes were the most common, representing $34.21 \%$ and $13.16 \%$ of all normal samples, respectively. $\mathrm{CCG}_{7}$ was the most common allele in both the HD and normal patients.

In normal chromosomes, the $(\mathrm{CCG})_{7}$ and $(\mathrm{CCG})_{10}$ alleles were associated with, on average, $17.6 \pm 3.94$ and $15.52 \pm 1.57$ CAG repeats, respectively. In HD chromosomes, the $(\mathrm{CCG})_{7}$ and $(\mathrm{CCG})_{10}$ alleles were associated with, on average, $42.52 \pm 4.52$ and $39.5 \pm 2.12 \mathrm{CAG}$ repeats, respectively.
Considering only the (CCG) 10 allele, our haplotype analysis showed that $(\mathrm{CCG})_{10}$ was linked to a CAG normal allele ( $<36$ CAG repeats) in 19 haplotypes $(90.48 \%)$ in a sample of 21 chromosomes.

Considering only the $(\mathrm{CCG})_{7}$ allele, $41.7 \%(n=40)$ of haplotypes had $(\mathrm{CCG})_{7}$ linked to an expanded CAG allele ( $>35$ CAG repeats) and $58.33 \%(n=56)$ had $(\mathrm{CCG})_{7}$ linked to a normal CAG allele $(<36$ CAG repeats) in a sample of 96 chromosomes.

Considering $(\mathrm{CCG})_{7}$ and $(\mathrm{CCG})_{10}$ alleles in haplotypes harboring the expanded CAG repeats, we found that $(\mathrm{CCG})_{7}$ was linked to expanded CAG repeats in 40 haplotypes (95.24\%), and (CCG) 10 was linked to expanded CAG repeats in only two haplotypes $(4.76 \%)$. We found a significant association between expanded CAG alleles and $(\mathrm{CCG})_{7}\left(\chi^{2}=6.97, P=0.0084\right)$. There was an association of $74 \%$ between DH-expanded alleles and the size of CCG alleles ((CCG $)_{7}$ and $(\mathrm{CCG})_{10}$ ), with odds ratio of 5.59 .

The mean age at HD onset was 43.28 years in individuals where the $(\mathrm{CCG})_{7}$ allele was associated with the expanded CAG repeat $(n=32)$. In two HD patients with $(\mathrm{CAG})_{41}-(\mathrm{CCG})_{10}$ or $(\mathrm{CAG})_{38}-(\mathrm{CCG})_{10}$ haplotypes, the ages at onset of the disease were 53 and 50 years, respectively.

\section{Inter- and intragenerational investigation of CAG repeats}

In eight of the twenty-one families studied, at least two individuals were molecularly tested. These eight families were used for the intragenerational survey (Figure 5). The intragenerational investigation of expanded CAG repeat alleles in siblings showed a variation from 0 to 5 CAG repeats, compared with the parental allele.

From these eight families, only four pedigrees were used for the intergenerational investigation because the molecularly tested subjects were parents and children. In these families, there were two paternally inherited and two maternally inherited expanded CAG repeats. In total, mothers transmitted three alleles with extra CAG repeats and one allele with fewer CAG repeats. By contrast, fathers transmitted four alleles with extra CAG repeats and no alleles with fewer CAG repeats.

In four cases of intergenerational transmission from the mother, we found that three individuals had extra CAG expansions and one individual had a contraction. Our intergenerational analysis showed that the number of CAG repeats increased from 1 to 8 between parent and child, with a mean increase of $2.75 \pm 2.63$ repeats. Maternally inherited CAG repeat expansion was found in three sons and one daughter. On the other hand, paternally inherited CAG repeat expansion was found in two daughters and two sons. Two families 
Table 1 CAG/CCG haplotypes in 122 chromosomes

\begin{tabular}{|c|c|c|c|}
\hline Haplotype number & Haplotype & Number of haplotypes & Frequency \\
\hline 2 & $(\mathrm{CAG}) 13-(\mathrm{CCG}) 7$ & 5 & 0.0410 \\
\hline 4 & (CAG)15-(CCG)7 & 26 & 0.2131 \\
\hline 5 & $(\mathrm{CAG}) 16$ - (CCG)7 & 4 & 0.0328 \\
\hline 6 & $(\mathrm{CAG}) 17-(\mathrm{CCG}) 7$ & 3 & 0.0246 \\
\hline 9 & $(\mathrm{CAG}) 20$-(CCG)7 & 2 & 0.0164 \\
\hline 10 & $(\mathrm{CAG}) 21-(\mathrm{CCG}) 7$ & 3 & 0.0246 \\
\hline 11 & $(C A G) 22-(C C G) 7$ & 2 & 0.0164 \\
\hline 12 & $(C A G) 23-(C C G) 7$ & 1 & 0.0082 \\
\hline 13 & (CAG)24-(CCG)7 & 1 & 0.0082 \\
\hline 14 & $(\mathrm{CAG}) 25-(\mathrm{CCG}) 7$ & 1 & 0.0082 \\
\hline 19 & $(\mathrm{CAG}) 39-(\mathrm{CCG}) 7$ & 6 & 0.0492 \\
\hline 20 & (CAG)40-(CCG)7 & 7 & 0.0574 \\
\hline 21 & $(\mathrm{CAG}) 41-(\mathrm{CCG}) 7$ & 4 & 0.0328 \\
\hline 22 & (CAG)42—(CCG)7 & 5 & 0.0410 \\
\hline 23 & $(\mathrm{CAG}) 43-(\mathrm{CCG}) 7$ & 3 & 0.0246 \\
\hline 24 & (CAG)45-(CCG)7 & 3 & 0.0246 \\
\hline 25 & $(\mathrm{CAG}) 46-(\mathrm{CCG}) 7$ & 2 & 0.0164 \\
\hline 26 & $(\mathrm{CAG}) 48-(\mathrm{CCG}) 7$ & 2 & 0.0164 \\
\hline 27 & $(\mathrm{CAG}) 49-(\mathrm{CCG}) 7$ & 1 & 0.0082 \\
\hline 28 & $(\mathrm{CAG}) 51-(\mathrm{CCG})_{7}$ & 1 & 0.0082 \\
\hline 29 & (CAG)53-(CCG)7 & 1 & 0.0082 \\
\hline 30 & (CAG)58-(CCG)7 & 1 & 0.0082 \\
\hline 38 & $(\mathrm{CAG}) 18-(\mathrm{CCG}) 10$ & 3 & 0.0246 \\
\hline 39 & $(\mathrm{CAG}) 38-(\mathrm{CCG}) 10$ & 1 & 0.0082 \\
\hline 40 & $(\mathrm{CAG}) 41-(\mathrm{CCG}) 10$ & 1 & 0.0082 \\
\hline Number of investigated chromosomes & & 122 & \\
\hline Haplotype number & Normal haplotype & Number of haplotypes & Frequency \\
\hline 2 & $(\mathrm{CAG}) 13-(\mathrm{CCG}) 7$ & 5 & 0.0658 \\
\hline 3 & $(\mathrm{CAG}) 14-(\mathrm{CCG}) 7$ & 2 & 0.0263 \\
\hline 4 & $(\mathrm{CAG}) 15-(\mathrm{CCG}) 7$ & 26 & 0.3421 \\
\hline 5 & $(\mathrm{CAG}) 16-(\mathrm{CCG}) 7$ & 4 & 0.0526 \\
\hline 6 & (CAG)17-(CCG)7 & 3 & 0.0395 \\
\hline 7 & (CAG)18-(CCG)7 & 1 & 0.0132 \\
\hline 8 & $(\mathrm{CAG}) 19-(\mathrm{CCG}) 7$ & 1 & 0.0132 \\
\hline 9 & $(\mathrm{CAG}) 20-(\mathrm{CCG}) 7$ & 2 & 0.0263 \\
\hline 10 & $(\mathrm{CAG}) 21-(\mathrm{CCG}) 7$ & 3 & 0.0395 \\
\hline 11 & $(\mathrm{CAG}) 22-(\mathrm{CCG}) 7$ & 2 & 0.0263 \\
\hline 12 & $(\mathrm{CAG}) 23-(\mathrm{CCG}) 7$ & 1 & 0.0132 \\
\hline 13 & $(\mathrm{CAG}) 24-(\mathrm{CCG}) 7$ & 1 & 0.0132 \\
\hline 14 & $(\mathrm{CAG}) 25-(\mathrm{CCG}) 7$ & 1 & 0.0132 \\
\hline 15 & $(\mathrm{CAG}) 26-(\mathrm{CCG}) 7$ & 1 & 0.0132 \\
\hline 16 & $(\mathrm{CAG}) 27-(\mathrm{CCG}) 7$ & 3 & 0.0395 \\
\hline 32 & $(\mathrm{CAG}) 15-(\mathrm{CCG}) 9$ & 1 & 0.0132 \\
\hline
\end{tabular}


Table 1 (Continued)

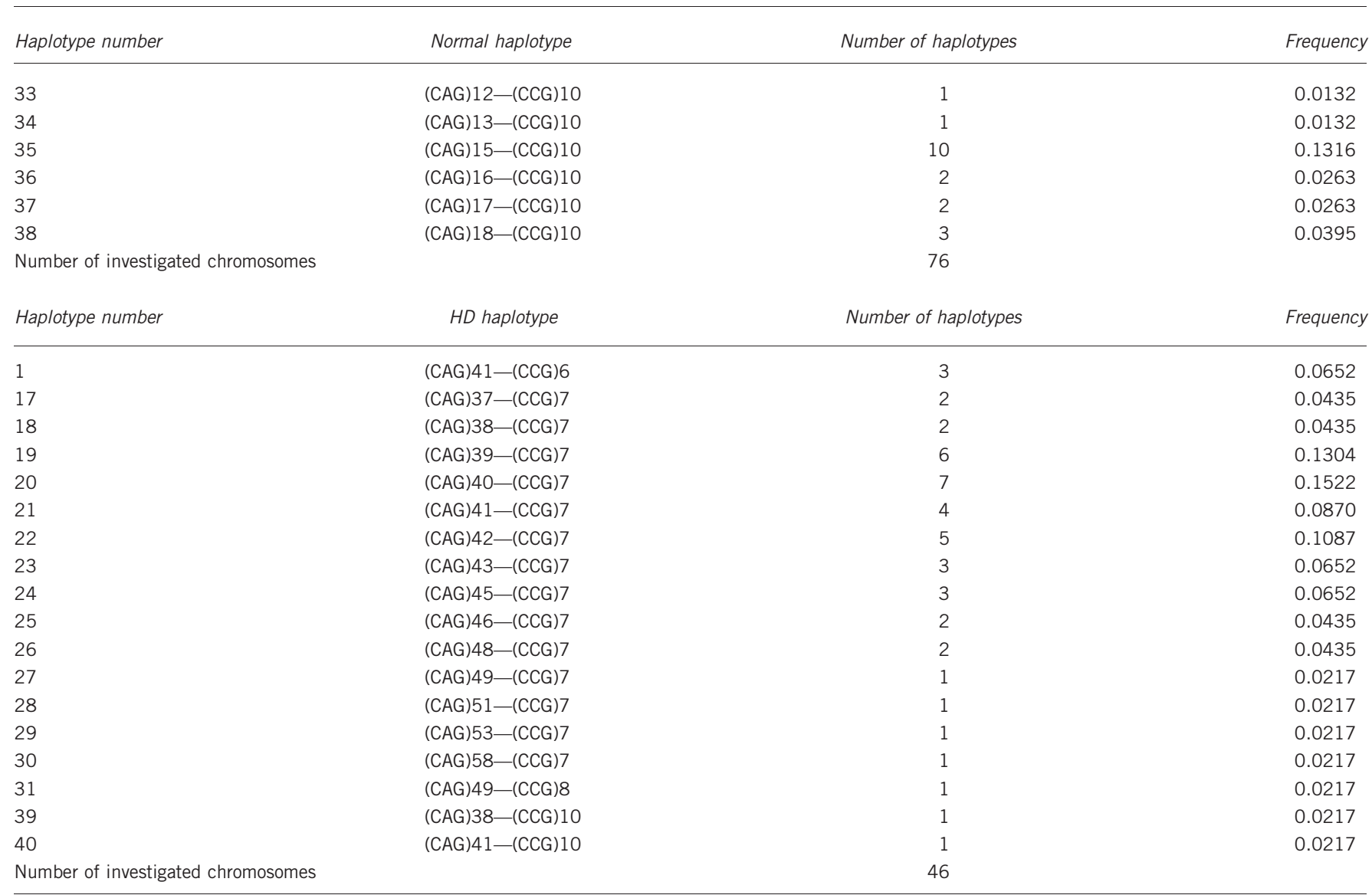

Separate tables of normal and expanded CAG alleles, ordered according to the size of CCG alleles.

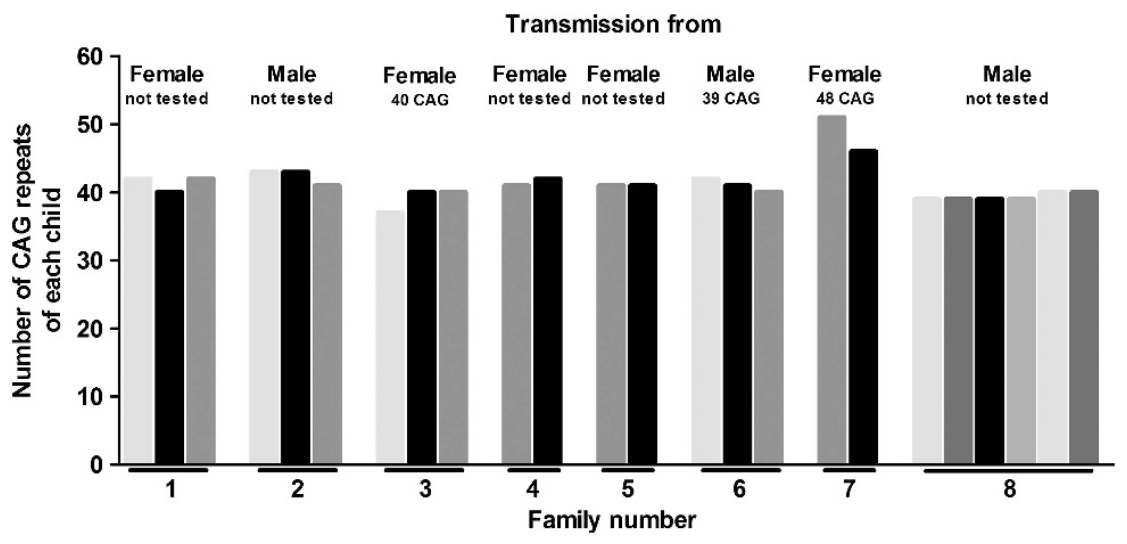

Figure 5 Transmission of CAG repeats from mothers (female) or fathers (male). Each bar indicates one family member.

showed a wider range of variation in the overall number of CAG repeats transmitted paternally. In the first of these two families, the father, who had 39 CAG repeats, transmitted alleles with $40(+1)$, $41(+2)$ and $42(+3)$ CAG repeats to his children. In the second family, the father had 38 CAG copies and transmitted an allele with 46 CAG repeats $(+8)$ to his offspring.

\section{DISCUSSION}

The rural city of Ervalia, in the state of Minas Gerais, has a high prevalence of HD (results not shown) because, historically, it was a place of social refuge for people with the disease and their families, who migrated in from more developed neighboring cities. As a result, the individuals in affected families who had immigrated to Ervália married within the community, without any genetic counselling; this led to the high prevalence of the disease. It is important to mention that, in the past, many patients from Ervália were misdiagnosed with Alzheimer's and Parkinson's disease, which contributed to misinformation among the risk group and led to an increase in cases in the city.

The clinical variability of the signs and symptoms of HD demands genetic investigation at molecular level for a definitive diagnosis. Furthermore, unstable expansions of CAG tracts have been found in a growing number of autosomal dominant degenerative disorders, 
including Kennedy disease and six types of autosomal dominant spinocerebellar ataxia (SCA): SCA1, SCA2, MJD/SCA3, SCA6, SCA7 and DRPLA. ${ }^{13}$

It is very difficult to define the smallest number of CAG repeats that cause the disease. Some authors consider alleles that bear 27 to 35 CAG repeats to be normal and non-mutable. ${ }^{13-15}$ These facts have generated confusion about the size of alleles responsible for causing HD.

Furthermore, it is known that somatic instability, which can lead to mosaicism of CAG repeats, can account for the HD phenotype in subjects carrying alleles with less than 36 CAG repeats. ${ }^{16,17}$

Clinical symptoms are always found in individuals with more than 39 CAG repeats. Thus, these alleles are classified as completely penetrant. ${ }^{10}$ The longest CAG repeat found by Seneca et al. ${ }^{18}$ was the repeat with $214 \mathrm{CAG}$ copies. Nance et al. ${ }^{19}$ reported an individual with juvenile-onset HD who had $250 \mathrm{CAG}$ repeats. This patient had a maternal history of $\mathrm{HD}$, but the mother's allele could not be determined because a DNA sample was not available.

In 2000, one survey of Brazilian HD patients showed that the normal alleles had 14 to 30 CAG repeats $($ mean $=18$; median 17$)$ and the expanded alleles had 39 to 88 repeats. ${ }^{14}$ Another survey of Brazilian patients found that the expanded alleles had 43 to 73 CAG repeats. ${ }^{13}$ Both the normal and the expanded alleles encountered in our investigation had fewer CAG repeats than those reported in previous Brazilian surveys. ${ }^{13,14}$ Although these three investigations focused on Brazilian populations, three ranges of CAG repeat sizes were found, which may reflect a sampling bias. However, it is important to mention that Brazil is a country of continental size and its five demographic regions (North, Northeast, Southeast, South and Central West) have populations with diverse origins, including Europe, Asia and Africa. Therefore, differences among the populations of distant geographic regions are expected. However, a larger sample size would be necessary for a significant statistical interpretation of the differences found in this analysis. Additionally, results are complicated by the fact environmental factors can also interfere with the variability of $\mathrm{HD}$ clinical manifestations. ${ }^{20}$

To our knowledge, this is the first investigation of the polymorphic CCG region and the CAG/CCG haplotypes in Brazil. The CCG polymorphic region may indicate the ancestral origin of the expanded CAG repeat allele that causes $\mathrm{HD},{ }^{21}$ given that they are located in the same chromosomal region, only $12 \mathrm{bp}$ apart. Each ethnic population shows a predominance of a certain number of CCG repeats that can vary from 6 to 12 repeats, ${ }^{22}$ with the exception of a case reported by Pramanik et al. ${ }^{23}$ in which four CCG repeats were identified.

The variability of the CAG allelic profile and the phenotypic aspects of HD in different populations have already been correlated with differing numbers of CCG repeats. ${ }^{15}$ We found that all of the chromosomes harboring HD-expanded CAG repeat alleles had CCG alleles with $6,7,8$ or 10 repeats and the most common allele was $(\mathrm{CCG})_{7}$ (Table 1). However, (CCG) 6 appeared in only two families that also showed $(\mathrm{CCG})_{7}$. In one of these families, an affected father who had a HD chromosome harboring $(\mathrm{CCG})_{7}$ had two affected daughters and one affected son, who had $(\mathrm{CCG})_{6},(\mathrm{CCG})_{7}$ and $(\mathrm{CCG})_{7}$ alleles on their HD chromosomes, respectively. These findings may indicate an intergenerational contraction of $(\mathrm{CCG})_{7}$ to $(\mathrm{CCG})_{6}$ in one daughter. The second family was not informative concerning the contraction, because the affected subjects were a pair of cousins whose parents had already died and were not tested.

Another family showed one affected subject with HD chromosome harboring $(\mathrm{CCG})_{8}$, who was a cousin of three brothers who had only
$(\mathrm{CCG})_{7}$ on their HD chromosomes. Their mothers were affected sisters, as well as their maternal grandfather. The three brothers' mother had a $(\mathrm{CCG})_{7}$ on her HD chromosome but the $(\mathrm{CCG})_{8}$ affected man's mother had died and was not tested. Therefore, we may only suspect that $(\mathrm{CCG})_{8}$ was an expansion of one CCG unit from $(\mathrm{CCG})_{7}$ within this family. Worldwide, the most common CCG alleles have 7 or 10 repeats. In Western Europe, $(C C G)_{7}$ is the most frequent allele. In populations with lower prevalence of $\mathrm{HD}$, such as those of Japanese and African descent, the most prevalent allele is (CCG) $10 \cdot{ }^{15,24}$ The potential correlation between the polymorphic CCG region and the age of onset of the disease is controversial. According to Panegyres et al. ${ }^{25}$ who studied an Australian population, the CCG region does not correlate with the age of onset of $\mathrm{HD}$, the neurological symptoms or the number of CAG repeats. On the other hand, Chattopadhyay et al. ${ }^{26}$ found that, in an Indian population, variation in the length of the CCG region was responsible for $3.1 \%$ of variation in the age of onset of the disease. We found no correlation between the number of CCG repeats and the age of onset of the disease $(r=0.06)$. By contrast, we found a significant negative correlation between the number of CAG repeats and the age of onset of the disease $(r=-0.84 ; P<0.001)$. This is consistent with other data in the literature, such as those of Wexler et al. ${ }^{20}(r=-0.6$; $P=0.0001$ ).

According to Costa et al., ${ }^{27}$ it is possible that the haplotypic environment of the CAG repeats in the HTT gene is of great importance in regard to the evolution and the origin of newly expanded chromosomes. In our study, the mean number of CAG repeats was higher in the normal chromosomes containing seven CCG repeats than in those with 10 CCG repeats. Furthermore, the association of CAG repeat size with $(\mathrm{CCG})_{7}$ in the HD chromosomes was very similar to that in the normal chromosomes. Similar results were also found in a Portuguese population. ${ }^{27}$

In our sample, as in others, ${ }^{22,23}$ the $(\mathrm{CCG})_{6}$ and $(\mathrm{CCG})_{8}$ alleles were rare. In Portuguese, Indian and Japanese populations, ${ }^{23,27,28}$ multiple CCG alleles were associated with expanded CAG repeats within individual families. These findings suggest that one mutational event may have led to these differences in the number of CCG repeats. ${ }^{29}$ The allelic diversity of CCG repeats is much lower than that of CAG repeats, indicating a lower overall mutation rate. ${ }^{30}$

An intergenerational Brazilian study ${ }^{14}$ found that five CAG alleles had a greater number of repeats (new expansions) and were inherited paternally, and two new expansions were inherited from the mother. On the other hand, only one CAG allele had fewer repeats (new contraction) and was inherited maternally. In two cases, the alleles did not vary intergenerationally. ${ }^{14}$

In our study, the transmitted CAG alleles had a mean change of 2.75 CAG repeats. This change is higher than that found in a previous study conducted in Venezuela, where the mean intergenerational change was 1.27 repeats. $^{31}$ However, these two estimates are not significantly different because of the relatively large standard deviations. In our survey, the mean size of the paternally inherited CAG alleles was $42.25 \pm 2.6$ repeats. A similar result was found in the Venezuelan kindred study, ${ }^{31}$ which showed a mean value of 44.4 CAG repeats.

The variability in the size of the transmitted CAG repeats was also investigated by Wheeler et al. ${ }^{31}$ in Venezuela relative to the gender of the siblings. In our study, the maternal transmission of longer expanded alleles was more frequently observed in sons than in daughters. These results are similar to that reported by Wheeler et al. ${ }^{31}$ We found that a greater variation in number of CAG occurred when the HD allele was paternally transmitted, and the only repeat contraction occurred when the mutation was maternally transmitted to a son. Wheeler et al. ${ }^{31}$ 
observed a greater number of repeat contractions when the allele was transmitted from mothers to daughters.

Other studies, ${ }^{31,32}$ which looked at additional expansion of CAG repeats within a generation (intergenerational studies), showed that there is a significant difference between the maternal and paternal inheritance of alleles with large CAG repeat expansions $(P<0.0001)$. Specifically, they found that fathers transmit longer expanded alleles more often than mothers do. On the other hand, repeat contractions are more frequently inherited from the mother. ${ }^{31,32}$

It is believed that the founding mutation for $\mathrm{HD}$ occurred in Western Europe and spread to other regions as a result of migration. In Western Europe, the (CCG) ${ }_{7}$ allele is predominant, and therefore, it has been hypothesized that independent mutational events led to variations in the number of CCG repeats. ${ }^{33,34}$

The mutational bias model for the evolution of HD predicts an ever-increasing incidence of HD. ${ }^{30}$ This model says that the allele frequency distributions we see today are not endpoints but a snapshot in an on-going process of gradual expansion. According to the model, the incidence of HD will accelerate because mutation towards detrimental repeat lengths happens over the course of several generations. ${ }^{30}$

Rubinsztein et al..$^{30}$ have speculated that single repeat units are gained and lost with equal frequency but that larger blocks are more likely to be gained rather than lost. This would provide a possible mechanism to link mutational bias, mutation rate and length dependency. ${ }^{30}$

Haplotype analysis demonstrated that certain normal chromosomes, with CAG lengths at the high range of normal, are prone to further expansion and eventually result in HD chromosomes. Analysis of normal chromosomes in different populations suggests that genetic factors contribute to CAG expansion and account for the worldwide variation in $\mathrm{HD}$ prevalence. ${ }^{35}$

In conclusion, the investigation of the CCG region and CAG/CCG haplotypes in this study were important because they focused on the Brazilian population, an understudied group. The expansion of CAG repeats was predominantly associated with the $\mathrm{CCG}_{7}$ allele. However, a more robust investigation, in conjunction with immigration and ethnic origin data, will be required to elucidate the ancestral origin of the HTT gene in Brazilians.

\section{CONFLICT OF INTEREST}

The authors declare no conflict of interest.

\section{ACKNOWLEDGEMENTS}

Many thanks are due to Professor LCS Thuler and G Tucher for helping with data analysis. We are grateful to CAPES/PROAP/PPGNEURO, FINEP, UNIRIO, CNPq and FAPERJ for the financial support, and to FAMINAS, Associação Brasil Huntington (ABH) and the Department of Genetics and Molecular Biology for referring cases to the clinical geneticists.

1 The Huntington's Disease Collaborative Research Group. A novel gene containing a trinucleotide repeat that is expanded and unstable on Huntington's disease chromosomes. Cell 72, 971-983 (1993).

2 Zuccato, C., Ciammola, A., Rigamonti, D., Leavitt, B. R., Goffredo, D., Conti, L. et al. Loss of Huntingtin-mediated BDNF gene transcription in Huntington's disease. Science 293, 493-498 (2001).

3 Andrew, S. E., Goldberg, Y. P., Kremer, B., Telenius, H., Theilmann, J., Adam, S. et al. The relationship between trinucleotide (CAG) repeat length and clinical features of Huntington's disease. Nat. Genet. 4, 398-403 (1993).

4 Nahhas, F. A., Garbern, J., Krajewski, K. M., Roa, B. B. \& Feldman, G. L. Juvenile onset Huntington disease resulting from a very large maternal expansion. Am. J. Med. Genet. A 137A, 328-331 (2005).
5 Beighton, P. \& Hayden, M. R. Huntington's chorea. S. Afr. Med. J. 59, 250 (1981).

6 Hayden, M. R., Goldblatt, J., Wallis, G., Winship, I. M. \& Beighton, P. Molecular genetics and Huntington's disease. The South African situation. S. Afr. Med. J 71, 683-686 (1987).

7 DiFiglia, M., Sapp, E., Chase, K. O., Davies, S. W., Bates, G. P., Vonsattel, J. P. et al. Aggregation of Huntingtin in neuronal intranuclear inclusions and dystrophic neurites in brain. Science 277, 1990-1993 (1997).

8 Gil, J. M. \& Rego, A. C. Mechanisms of neurodegeneration in Huntington's disease. Eur. J. Neurosci 27, 2803-2820 (2008).

9 Bonelli, R. M. \& Hofmann, P. A systematic review of the treatment studies in Huntington's disease since 1990. Expert Opin. Pharmacother. 8, 141-153 (2007).

10 ACMG/ASHG Statement. Laboratory guidelines for Huntington disease genetic testing. The American College of Medical Genetics/American Society of Human Genetics Huntington Disease Genetic Testing Working Group. Am. J. Hum. Genet. 62, 1243-1247 (1998).

11 Woolf, B. On estimating the relation between blood group and disease. Ann. Hum. Genet. 19, 251-253 (1955).

12 Haldane, J. B. S. \& Smith, SM. The sampling distribution of a maximum likelihood estimate. Biometrika 43, 96-103 (1956)

13 Lima, E. S. T. C., Serra, H. G., Bertuzzo, C. S. \& Lopes-Cendes, I. Molecular diagnosis of Huntington disease in Brazilian patients. Arq Neuropsiquiatr. 58, 11-17 (2000).

14 Raskin, S., Allan, N., Teive, H. A., Cardoso, F., Haddad, M. S., Levi, G. et al. Huntington disease: DNA analysis in Brazilian population. Arq Neuropsiquiatr. 58, 977-985 (2000)

15 Morovvati, S., Nakagawa, M., Osame, M. \& Karami, A. Analysis of CCG repeats in Huntingtin gene among HD patients and normal populations in Japan. Arch. Med. Res. 39, 131-133 (2008).

16 Andrich, J., Arning, L., Wieczorek, S., Kraus, P. H., Gold, R. \& Saft, C. Huntington's disease as caused by 34 CAG repeats. Mov. Disord. 23, 879-881 (2008).

17 Telenius, H., Kremer, B., Goldberg, Y. P., Theilmann, J., Andrew, S. E., Zeisler, J. et al. Somatic and gonadal mosaicism of the Huntington disease gene CAG repeat in brain and sperm. Nat. Genet. 6, 409-414 (1994).

18 Seneca, S., Fagnart, D., Keymolen, K., Lissens, W., Hasaerts, D., Debulpaep, S. et al. Early onset Huntington disease: a neuronal degeneration syndrome. Eur. J. Pediatr. $163,717-721$ (2004)

19 Nance, M. A., Mathias-Hagen, V., Breningstall, G., Wick, M. J. \& McGlennen, R. C. Analysis of a very large trinucleotide repeat in a patient with juvenile Huntington's disease. Neurology 52, 392-394 (1999).

20 Wexler, N. S., Lorimer, J., Porter, J., Gomez, F., Moskowitz, C., Shackell, E. et al. Venezuelan kindreds reveal that genetic and environmental factors modulate Huntington's disease age of onset. Proc. Natl Acad. Sci. USA 101, 3498-3503 (2004).

21 Muglia, M., Leone, O., Annesi, G., Gabriele, A. L., Imbrogno, E., Grandinetti, C. et al. Nonisotopic method for accurate detection of (CAG)n repeats causing Huntington disease. Clin. Chem. 42, 1601-1603 (1996).

22 Squitieri, F., Andrew, S. E., Goldberg, Y. P., Kremer, B., Spence, N., Zeisler, J. et al. DNA haplotype analysis of Huntington disease reveals clues to the origins and mechanisms of CAG expansion and reasons for geographic variations of prevalence. Hum. Mol. Genet. 3, 2103-2114 (1994).

23 Pramanik, S., Basu, P., Gangopadhaya, P. K., Sinha, K. K., Jha, D. K., Sinha, S. et al. Analysis of CAG and CCG repeats in Huntingtin gene among HD patients and normal populations of India. Eur. J. Hum. Genet. 8, 678-682 (2000).

24 Hecimovic, S., Klepac, N., Vlasic, J., Vojta, A Janko, D., Skarpa-Prpic, I. et al. Genetic background of Huntington disease in Croatia: molecular analysis of CAG, CCG, and Delta2642 (E2642del) polymorphisms. Hum. Mutat. 20, 233 (2002).

25 Panegyres, P. K., Beilby, J., Bulsara, M., Toufexis, K. \& Wong, C. A study of potential interactive genetic factors in Huntington's disease. Eur. Neurol. 55, 189-192 (2006).

26 Chattopadhyay, B., Ghosh, S., Gangopadhyay, P. K., Das, S. K., Roy, T., Sinha, K. K. et al. Modulation of age at onset in Huntington's disease and spinocerebellar ataxia type 2 patients originated from eastern India. Neurosci. Lett. 345, 93-96 (2003)

27 Costa, M. C., Magalhaes, P., Guimaraes, L., Maciel, P., Sequeiros, J. \& Sousa, A. The $\mathrm{CAG}$ repeat at the Huntington disease gene in the Portuguese population: insights into its dynamics and to the origin of the mutation. J. Hum. Genet. 51, 189-195 (2006).

28 Masuda, N., Goto, J., Murayama, N., Watanabe, M., Kondo, I. \& Kanazawa, I. Analysis of triplet repeats in the Huntingtin gene in Japanese families affected with Huntington's disease. J. Med. Genet. 32, 701-705 (1995).

29 Wang, C. K., Wu, Y. R., Hwu, W. L., Chen, C. M., Ro, L. S., Chen, S. T. et al. DNA haplotype analysis of CAG repeat in Taiwanese Huntington's disease patients. Eur. Neurol. 52, 96-100 (2004)

30 Rubinsztein, D. C., Amos, W., Leggo, J., Goodburn, S., Ramesar, R. S., Old, J. et al. Mutational bias provides a model for the evolution of Huntington's disease and predicts a general increase in disease prevalence. Nat. Genet. 7, 525-530 (1994).

31 Wheeler, V. C., Persichetti, F., McNeil, S. M., Mysore, J. S., Mysore, S. S., MacDonald, M. E. et al. Factors associated with HD CAG repeat instability in Huntington disease. J. Med. Genet. 44, 695-701 (2007).

32 Kovtun, I. V., Spiro, C. \& McMurray, C. T. Triplet repeats and DNA repair: germ cell and somatic cell instability in transgenic mice. Methods Mol. Biol. 277, 309-319 (2004).

33 Hayden, M. R., Berkowicz, A. L., Beighton, P. H. \& Yiptong, C. Huntington's chorea on the island of Mauritius. S. Afr. Med. J 60, 1001-1002 (1981)

34 Garcia-Planells, J., Burguera, J. A., Solis, P., Millan, J. M., Ginestar, D., Palau, F. et al. Ancient origin of the CAG expansion causing Huntington disease in a Spanish population. Hum. Mutat. 25, 453-459 (2005).

35 Andrew, S. E. \& Hayden, M. R. Origins and evolution of Huntington disease chromosomes. Neurodegeneration 4, 239-244 (1995). 